\title{
Logarithmic relaxation in a kinetically constrained model
}

\author{
Angel J. Moreno ${ }^{\text {a) }}$ \\ Donostia International Physics Center, Paseo Manuel de Lardizabal 4, 20018 San Sebastián, Spain \\ Juan Colmenero \\ Donostia International Physics Center, Paseo Manuel de Lardizabal 4, 20018 San Sebastián, Spain; \\ Departamento de Física de Materiales, Universidad del País Vasco (UPV/EHU), Apartado 1072, \\ 20080 San Sebastián, Spain; and Unidad de Física de Materiales, Centro Mixto CSIC-UPV, \\ Apartado 1072, 20080 San Sebastián, Spain
}

(Received 6 April 2006; accepted 17 May 2006; published online 5 July 2006)

[DOI: $10.1063 / 1.2212422]$

Very recently, we have reported a numerical investigation on a simple bead-spring model for polymer blends with components of very different relaxation times. ${ }^{1}$ At moderate and high dilution of the fast component, the slow component acts as a confining medium for the former. In this situation, dynamic correlators for the fast component display nonstandard relaxation features. Instead of the usual two-step decay observed for dense colloidal systems or deeply supercooled liquids, dynamic correlators do not exhibit a defined plateau and the long-time decay is highly stretched. Decreasing temperature yields a striking concave-to-convex crossover in the shape of the correlators. At some intermediate temperature, the decay is purely logarithmic over three to four time decades. Atomistic simulations on real polymer blends show a qualitatively similar behavior. ${ }^{2}$ These results exhibit striking analogies with predictions of the mode coupling theory (MCT) for state points close to a higher-order transition. ${ }^{3,4} \mathrm{In}$ particular, asymptotic laws predicted by this MCT scenario for dynamic correlators provide an excellent description of simulation results for the fast component. ${ }^{1}$

Higher-order MCT transitions have been strictly derived for simple models of short-ranged attractive colloids. ${ }^{4-7}$ For these systems, such transitions arise in regions of the control parameter space where reversible bond formation (originating from the short-ranged attraction) and hard-sphere repulsion compete as different mechanisms for dynamic arrest. The observed strong dynamic analogies with polymer blends ${ }^{1}$ suggest that this anomalous relaxation scenario might be a general feature of systems where dynamic arrest is driven by several competing mechanisms of different characteristic lengths. For the fast component in polymer blends we have suggested confinement and bulklike caging, ${ }^{1}$ respectively, induced by the host matrix formed by the slow component, and by neighboring particles of the fast component.

Kinetically constrained models ${ }^{8,9}$ are used as coarsegrained pictures for relaxation in supercooled liquids. In these models, the liquid structure is substituted by an array of cells. The cell size roughly corresponds to a density correlation length. A spin variable is assigned to each cell, with values 0 or 1 denoting, respectively, unexcited and excited local states in a mobility field. Change in local mobility (spin flip) for a given cell is permitted according to kinetic constraints determined by the mobilities of neighboring cells.
Propagation of mobility, leading to structural relaxation, occurs via dynamic facilitation: microscopic regions become temporarily mobile only if neighboring regions are mobile. Kinetically constrained models provide in a simple way an important feature of glass-forming liquids: the increasing of dynamical correlation lengths with decreasing temperature, leading to dynamic heterogeneity. ${ }^{10-13}$

Some of the anomalous dynamic features displayed by short-ranged attractive colloids, as logarithmic relaxation or reentrant behavior of the diffusivity, have been recently reproduced by a simple model based on this latter approach. ${ }^{14}$ Motivated by this fact and by the mentioned dynamic analogies between short-ranged attractive colloids and polymer blends, in this Note we investigate a simple kinetically constrained model aimed to reproduce dynamic features in real binary systems with components of very different relaxation rates. Results reported here exhibit strong analogies with dynamic correlators in polymer blends.

We have carried out Monte Carlo (MC) simulations on a variation (see below) of the north-or-east-or-front (NEF) model recently investigated by Berthier and Garrahan. ${ }^{13}$ In this three-dimensional model, spin flip of a given cell is only permitted if there is at least one excited neighboring cell in the north-or-east-or-front direction. ${ }^{15}$ More specifically, if the cell is denoted by its position vector $\mathbf{i}=\left(i_{x}, i_{y}, i_{z}\right)$, spin flip is permitted according to the following rules.

(i) At least one of the neighboring cells in the north $\left(i_{x}, i_{y}, i_{z}+1\right)$, east $\left(i_{x}, i_{y}+1, i_{z}\right)$, or front $\left(i_{x}+1, i_{y}, i_{z}\right)$ direction is excited, i.e., it has spin 1.

(ii) If (i) is fulfilled, spin flip is accepted according to the Metropolis rule. ${ }^{16}$ Hence, $1 \rightarrow 0$ is always accepted, while $0 \rightarrow 1$ is accepted with a thermal probability $[1+\exp (1 / T)]^{-1}$, where $T$ is the temperature.

In the present work we investigate a binary mixture of cells with the same population of excitations, $[1+\exp (1 / T)]^{-1}$, but different rates for excitation, $\exp (-E / T)[1+\exp (1 / T)]^{-1}$, and de-excitation, $\exp (-E / T)$, of cell mobility. This choice fulfills detailed balance. We use $E=0$ and 3 for the different components, which are, respectively, denoted as "fast" and "slow" cells. Hence, spin flip rules for the fast component are the same as in the original NEF model, aimed to reproduce bulklike dynamics. The 


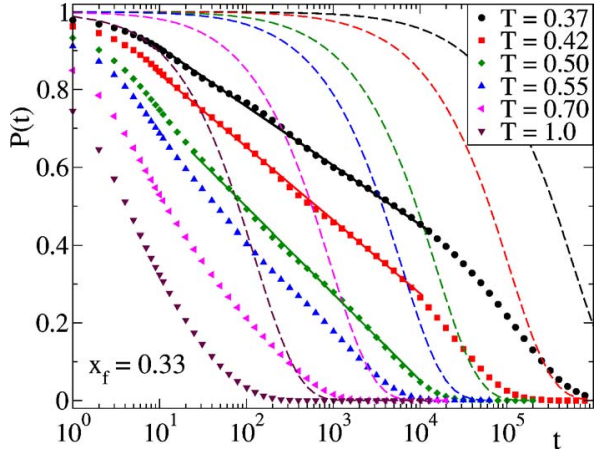

FIG. 1. Temperature dependence of the mean persistence function $P(t)$ at composition $x_{f}=0.33$. The symbols correspond to fast cells. The dashed lines correspond to slow cells (same temperatures as for the former ones, decreasing from left to right). The solid straight lines indicate nearly logarithmic relaxation.

probability of spin flip for slow cells is decreased by a factor $\exp (-3 / T)$ as compared to that of fast cells, providing a large separation in the time scales of both components (see below).

The mixture composition, $x_{f}$, is defined as the fraction of fast cells. We investigate a wide range of composition and temperature as control parameters. A square box of side $N$ $=50$ cells is used . Periodic boundary conditions are implemented. Slow and fast cells are randomly distributed according to the selected value of $x_{f}$. Time is given in MC steps. Within each MC step a total of $N^{3}$ trials (one for each cell) are performed.

We have evaluated the mean persistence function $P(t)$ $=\Sigma_{\mathrm{i}} p(\mathbf{i} ; t)$, where $p(\mathbf{i} ; t)$ is the persistence function of the cell $\mathbf{i}$ at time $t$. It takes the value 1 if no spin flip has occurred for that cell in the interval $[0, t]$. If one or more spin flips have occurred, it takes the value 0. Figure 1 shows results for $P(t)$ at composition $x_{f}=0.33$ and different temperatures. Note that the first decay usually observed for any correlator in real systems - corresponding to the transient regime at microscopic times-is obviously absent due to the coarse-grained character of the model. The introduction of very different rates for cell spin flip provides very different relaxation times for fast and slow cells. Slow cells display standard relaxation, as observed for the slow component in polymer blends. ${ }^{1}$ However, fast cells display rather different relaxation features. In complete analogy with the results for the fast component in polymer blends, ${ }^{1,2} P(t)$ exhibits a concave-to-convex crossover with decreasing temperature. At an intermediate temperature, pure logarithmic relaxation is observed over a time interval of near three decades. This behavior is also observed by fixing temperature and varying the mixture composition (see Fig. 2). It is worthy of remark that the features displayed in Figs. 1 and 2 are not specific of $P(t)$. Qualitatively analogous results are exhibited by other

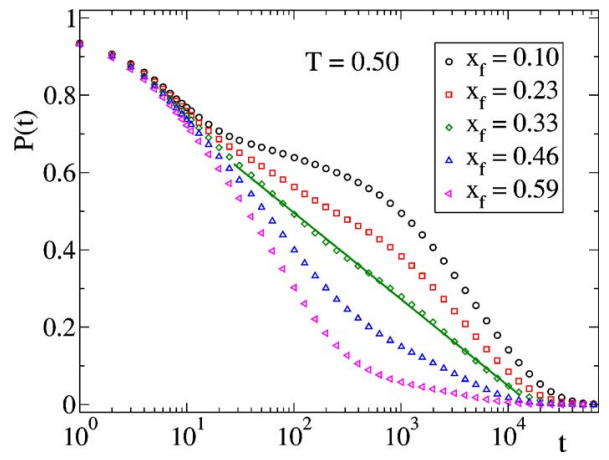

FIG. 2. Symbols: composition dependence of $P(t)$, at $T=0.50$, for the fast cells. The straight line indicates nearly logarithmic relaxation.

dynamic correlators, as persistence correlators for distinct cells, or intermediate scattering functions. ${ }^{17}$

In summary, the highly nontrivial anomalous relaxation features recently reported for the fast component in polymer blends can be qualitatively reproduced by a simple kinetically constrained model. The fact that another recent work within this approach ${ }^{14}$ also reproduces unusual relaxation features for short-ranged attractive colloids suggest the relevance of the dynamic facilitation picture for systems with several competing mechanisms for dynamic arrest.

We thank J. P. Garrahan and L. Berthier for useful discussions.

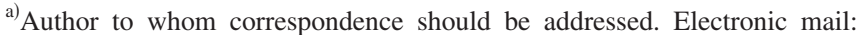
wabmosea@sq.ehu.es

${ }^{1}$ A. J. Moreno and J. Colmenero, J. Chem. Phys. 124, 184906 (2006).

${ }^{2}$ A. C. Genix, A. Arbe, F. Alvarez, J. Colmenero, L. Willner, and D. Richter, Phys. Rev. E 72, 031808 (2005); J. Colmenero, A. J. Moreno, A. C. Genix, A. Arbe, and F. Alvarez (unpublished).

${ }^{3}$ W. Götze and R. Haussmann, Z. Phys. B: Condens. Matter 72, 403 (1988); W. Götze and M. Sperl, Phys. Rev. E 66, 011405 (2002).

${ }^{4}$ M. Sperl, Phys. Rev. E 68, 031405 (2003).

${ }^{5}$ L. Fabbian, W. Götze, F. Sciortino, P. Tartaglia, and F. Thiery, Phys. Rev. E 59, R1347 (1999).

${ }^{6}$ J. Bergenholtz and M. Fuchs, Phys. Rev. E 59, 5706 (1999).

${ }^{7}$ K. Dawson, G. Foffi, M. Fuchs, W. Götze, F. Sciortino, M. Sperl, P. Tartaglia, T. Voigtmann, and E. Zaccarelli, Phys. Rev. E 63, 011401 (2000).

${ }^{8}$ G. H. Fredrickson and H. C. Andersen, Phys. Rev. Lett. 53, 1244 (1984).

${ }^{9}$ P. Sollich and M. R. Evans, Phys. Rev. Lett. 83, 3238 (1999).

${ }^{10}$ J. P. Garrahan and D. Chandler, Proc. Natl. Acad. Sci. U.S.A. 100, 9710 (2003).

${ }^{11}$ J. P. Garrahan and D. Chandler, Phys. Rev. Lett. 89, 035704 (2002).

${ }^{12}$ L. Berthier and J. P. Garrahan, Phys. Rev. E 68, 041201 (2003).

${ }^{13}$ L. Berthier and J. P. Garrahan, J. Phys. Chem. B 109, 3578 (2005).

${ }^{14}$ P. L. Geissler and D. R. Reichman, Phys. Rev. E 71, 031206 (2005).

${ }^{15}$ Directionality in the kinetic constraints is imposed to mimic fragile liquid behavior (Ref. 13).

${ }^{16}$ D. Frenkel and B. Smit, Understanding Molecular Simulation (Academic, San Diego, 1996).

${ }^{17}$ For a definition of intermediate scattering functions in kinetically constrained models see, e.g., D. Chandler, J. P. Garrahan, R. L. Jack, L. Maibaum, and A. C. Pan, e-print cond-mat/0605084. 